\title{
The feasibility, reliability, and incremental value of two-dimensional speckle-tracking for the detection of significant coronary stenosis after treadmill stress echocardiography
}

Marc-André d'Entremont ${ }^{1,2^{*}}$, Gabriel Fortin ${ }^{1}$, Thao Huynh ${ }^{3}$, Étienne Croteau ${ }^{4}$, Paul Farand ${ }^{1}$, Samuel Lemaire-Paquette ${ }^{4}$, Marie-Claude Brochu' ${ }^{1}$ Doan Hoa Do ${ }^{1}$, Serge Lepage ${ }^{1}$, Warner Mbuila Mampuya ${ }^{1}$, Étienne L. Couture ${ }^{1}$, Michel Nguyen ${ }^{1}$ and Btissama Essadiqi ${ }^{1}$

\begin{abstract}
Background: Two-dimensional speckle-tracking echocardiography (STE) may help detect coronary artery disease (CAD) when combined with dobutamine stress echocardiography. However, few studies have explored STE with exercise stress echocardiography (ESE). We aimed to evaluate the feasibility, reliability, and incremental value of STE combined with treadmill ESE compared to treadmill ESE alone to detect CAD.

Methods: We conducted a case-control study of all consecutive patients with abnormal ESE in 2018-2020 who subsequently underwent coronary angiography within a six-month interval. We 1:1 propensity score-matched these patients to those with a normal ESE. Two blinded operators generated a 17-segment bull's-eye map of longitudinal strain (LS). We utilized the mean differences between stress and baseline LS values in segments 13-17, segment 17, and segments 15-16 to create receiver operator curves for the overall examination, the left anterior descending artery $(L A D)$, and the non-LAD territories, respectively.

Results: We excluded 61 STEs from 201 (30.3\%) eligible ESEs; 47 (23.4\%) because of suboptimal image quality and 14 (7.0\%) because of excessive heart rate variability precluding the calculation of a bull's-eye map. After matching, a total of 102 patients were included (51 patients in each group). In the group with abnormal ESE patients (mean age 66.4 years, $39.2 \%$ female), $64.7 \%$ had significant CAD (> 70\% stenosis) at coronary angiogram. In the group with normal ESE patients (mean age 65.1 years, 35.3\% female), 3.9\% were diagnosed with a new significant coronary stenosis within one year. The intra-class correlation for global LS was 0.87 at rest and 0.92 at stress, and 0.84 at rest, and 0.89 at stress for the apical segments. The diagnostic accuracy of combining ESE and STE was superior to visual assessment alone for the overall examination (area under the curve (AUC) $=0.89$ vs. $0.84, p=0.025$ ), the non-LAD territory ( $A \cup C=0.83$ vs. $0.70, p=0.006$ ), but not the LAD territory ( $A \cup C=0.79$ vs. $0.73, p=0.11$ ).
\end{abstract}

Conclusions: Two-dimensional speckle-tracking combined with treadmill ESE is relatively feasible, reliable, and may provide incremental diagnostic value for the detection and localization of significant CAD.

\footnotetext{
*Correspondence: marc-andre.dentremont@usherbrooke.ca

${ }^{1}$ Division of Cardiology, Department of Medicine, Sherbrooke University

Hospital Center (CHUS), 3001, 12e Avenue Nord, Sherbrooke, QC J1H 5N4,

Canada

Full list of author information is available at the end of the article
}

(c) The Author(s) 2021. Open Access This article is licensed under a Creative Commons Attribution 4.0 International License, which permits use, sharing, adaptation, distribution and reproduction in any medium or format, as long as you give appropriate credit to the original author(s) and the source, provide a link to the Creative Commons licence, and indicate if changes were made. The images or other third party material in this article are included in the article's Creative Commons licence, unless indicated otherwise in a credit line to the material. If material is not included in the article's Creative Commons licence and your intended use is not permitted by statutory regulation or exceeds the permitted use, you will need to obtain permission directly from the copyright holder. To view a copy of this licence, visit http://creativecommons.org/licenses/by/4.0/. The Creative Commons Public Domain Dedication waiver (http://creativeco mmons.org/publicdomain/zero/1.0/) applies to the data made available in this article, unless otherwise stated in a credit line to the data. 
Keywords: Coronary artery disease, Exercise stress echocardiography, Speckle-tracking, Strain-imaging

\section{Introduction}

Exercise stress echocardiography (ESE) is a well-validated diagnostic method for detecting coronary artery disease (CAD) [1]. However, substantial expertise and experience are required to achieve optimal diagnostic accuracy [2]. Furthermore, a significant proportion of false-positive ESEs may occur, resulting in unnecessary normal coronary angiograms $[3,4]$. The development of practical quantitative analysis techniques to increase the diagnostic yield of ESE has been a primary research focus [1]. Two-dimensional speckle-tracking echocardiography (STE), a quantitative measure of myocardial deformation, has been a recently developed method under investigation for the detection of CAD [5].

STE has not reliably demonstrated an increased accuracy compared to visual assessment for the detection of CAD with dobutamine stress echocardiography (DSE) [1, 6-8]. While researchers have explored the combination of STE with exercise stress echocardiography (ESE) with bicycle protocols, few have explored the combination with a treadmill protocol [9-11]. Furthermore, several studies that reported promising results of STE as a potential diagnostic technique have used unmatched controls [12-15]. As age, sex, diabetes, hypertension, smoking, and metabolic syndrome influence STE values, these studies may have been confounded by a higher burden of comorbidities in patients with CAD [16-20]. In addition, numerous studies have excluded patients with known CAD and baseline wall motion abnormalities (WMA), reducing the findings' potential generalizability $[15,21]$.

The majority of STE studies in DSE have used the global longitudinal strain (GLS) as a potential marker of ischemia [1]. However, the apical segments may be of greater interest because they typically display ischemic changes regardless of the location of the coronary stenosis [1]. Furthermore, a previous study suggested larger rest and stress variability in STE values in apical segments than mid-ventricular and basal segments, possibly increasing discrimination for significant coronary stenoses [22].

There is a need for studies that validate STE use in treadmill ESE. We aimed to conduct a pilot study to foremost evaluate the feasibility and interobserver reliability for STE in treadmill ESE. Secondly, as a hypothesis-generating analysis, we explored the incremental value of STE of the apical segments combined with the visual analysis of treadmill ESE compared to treadmill ESE alone to reclassify false-positive treadmill ESE.

\section{Material and methods Population and study design}

We conducted a case-control study between March 2018 and March 2020. We included all consecutive patients with an abnormal ESE between March 2018 and March 2020 who subsequently underwent a coronary angiogram within a 6-month interval for the investigation of obstructive CAD at the Sherbrooke University Hospital Center (CHUS) in Quebec, Canada.

We included patients who had reached at least $85 \%$ of their maximum predicted heart rate with a normal ESE for the control group. Normal ESE controls were only selected from March 2018 to August 2018 to allow for a one-year ascertainment period. Therefore, patients with normal ESE from August 2018 to March 2020 were excluded. We ascertained new significant coronary artery stenoses by non-invasive testing (stress EKG, exercise stress echocardiogram, dobutamine stress echocardiogram or nuclear myocardial perfusion imaging) and coronary angiogram after the normal ESE using the hospital's electronic health records. Deaths were recorded as a competing risk. Controls who were diagnosed with a new coronary stenosis within one year despite a normal ESE were considered false negatives.

We only used the first ESE per patient for both groups and excluded all patients who underwent DSE, those who required contrast agent for ESE, and those with prior coronary artery bypass grafting (CABG). Of note, all patients with previously diagnosed $C A D$ required a diagnosis by coronary angiogram and required subsequent complete percutaneous revascularization (all residual stenoses $\leq 50 \%$ ) to allow for ascertainment of new significant coronary stenoses during follow-up. The study was approved by the Sherbrooke University Hospital Research Center (CRCHUS) ethics committee, and individual patient consent was not required.

\section{Treadmill exercise stress echocardiography}

We performed ESE as per the recommendations of the American Heart Association (AHA) and the American Society of Echocardiography (ASE) guidelines [1, 23]. Images were obtained by five experienced sonographers using the GE Vivid E95 and M5Sc transducer (GE Vingmed Ultrasound AS, Horten, Norway) at baseline and within $90 \mathrm{~s}$ post-exercise with patients in the left lateral decubitus position. We recorded standard $2 \mathrm{D}$ grayscale images for the assessment of regional wall thickening in the apical four-chamber, apical two-chamber, apical longaxis, parasternal long- and short-axis views. We stored 
cine images of three representative cardiac cycles of the three apical views at baseline and post-exercise on an external hard drive for future analysis. The mean frame rate for all ESE was $48.7 \pm 9$ frames/sec.

Wall motion was evaluated at the time of examination by one of the six experienced echocardiographers in our laboratory. As the wall motion analysis was performed before angiography and STE, the echocardiographers were blinded to all other imaging results. Visual analysis was done at baseline and stress using all views and the 16 -segment model as per guideline recommendations. ${ }^{1}$ We considered any stress-induced WMA as an abnormal result. In patients with baseline WMA, akinesis that became dyskinesis was not considered as an abnormal result as this generally corresponds to the mechanical response of an infarcted segment rather than ischemia [1]. Furthermore, patients with baseline WMA were considered as having a normal ESE if no additional WMA were observed during ESE.

\section{Two-dimensional speckle-tracking strain analysis}

Two operators (MAD, GF), blinded to all other imaging results, performed $2 \mathrm{D}$ speckle-tracking on all eligible ESE using the automated function imaging (AFI) stress software version 201 (GE Vingmed Ultrasound AS, Horten, Norway) directly on the GE Vivid E95. The first operator (MAD) was a first-year general cardiology fellow who underwent formal echocardiography training and completed a ten-patient STE training set under the supervision of a senior echocardiographer (BE). The second operator (GF) was a final-year general cardiology fellow with level II certification. We manually traced the endocardial borders at the end-systolic frame in the three apical views at baseline and peak stress while optimizing the region of interest to better capture the myocardium. We defined the timing of aortic valve closure using the apical long-axis view. AFI software was then used to track the myocardial deformation and reject the poorly captured segments. We readjusted the endocardial tracing in the presence of poor tracking. We excluded all patients whose ESE had more than two rejected segments overall or any rejected apical segments at rest or stress. We also excluded all patients whose largest heart rate difference between two of the three apical images exceeded $30 \%$, as this precluded the software from calculating the bull's-eye. Notably, the AFI software was unable to calculate the STE in the presence of a contrast agent.

The software automatically calculated a 17 -segment bull's-eye map and GLS. We defined segments 1, 2, 7, 8, and 13-17 as corresponding to the left anterior descending (LAD) artery, and segments 3, 4, 5, 6, and 9-12 as corresponding to the non-LAD territory using the standard template of coronary anatomy (Fig. 1) [1, 5]. Using the apical segments exclusively, we also defined segments 13-17 for the overall examination; segment 17 as corresponding to the LAD; and segments $15-16$ as corresponding to the non-LAD territory. As we included patients with baseline WMA, we also calculated the delta strain values for standardization by subtracting the baseline values from the stress values. Stenosis of the left main coronary artery was considered to involve both the $\mathrm{LAD}$ and the circumflex coronaries.

\section{Coronary angiogram}

An experienced interventional cardiologist interpreted the images from the diagnostic coronary angiogram. We defined significant $C A D$ as $\geq 70 \%$ luminal diameter stenosis in at least one of the coronary arteries or as $\geq 50 \%$ in the left main coronary artery measured in the worstview angiographic projection [24].

\section{Statistical analysis}

To control for imbalances in baseline patient characteristics, we calculated propensity scores as the predicted probability of having an abnormal ESE using a multivariable logistic regression model. The covariates were chosen using a priori subject matter knowledge and included age, sex, hypercholesterolemia, hypertension, diabetes mellitus, current smoking status, and prior diagnosis of CAD. We matched 1:1 all patients with abnormal ESE to a normal ESE control using the optimal matching algorithm [25]. We assessed the covariate balance between the abnormal and normal ESE groups before and after propensity score matching using standardized differences [26].

We compared the clinical and ESE characteristics. Continuous data with normal distribution were

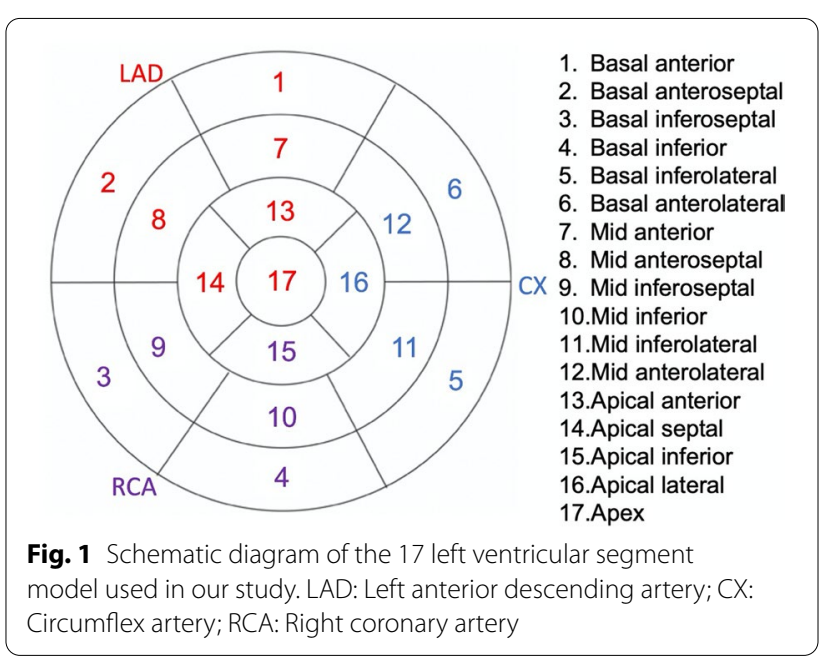


presented as means with standard deviations and analyzed using the Student's t-test. Non-normal data were presented as medians with interquartile ranges and compared using the Wilcoxon rank-sum test. We performed chi-square tests for dichotomous variables unless the expected number of frequencies was less than 5, in which case we used a Fisher's exact test. We used a two-sided significance level of 0.05 .

Interobserver variability in GLS and apical strain was determined by calculating the Pearson correlation coefficient, bias, limit of agreement, mean relative difference, and mean intra-class correlation coefficient. We calculated the mean intra-class correlation (ICC) using a two-way mixed-effect model measuring absolute agreement based on the mean rating [27].

We created receiver operating characteristic (ROC) curves to compare the diagnostic performance of STE and visual assessment. We used the mean between both operators for all strain measurements. Optimal cut-offs for sensitivity and specificity were determined using Youden's
J statistic. For patients with abnormal ESE, the coronary angiogram was used as the gold standard. Patients with normal ESE were considered positive if they had a newly diagnosed significant coronary stenosis in the one-year ascertainment period, and negative otherwise.

To determine the incremental value of STE to reclassify the false-positive ESE, we considered a visually normal ESE to be negative. As a second step, we then used STE to further classify the abnormal ESEs as positive or not for significant CAD using the coronary angiograms as the gold standard. When combined with visual assessment, the optimal cut-offs of STE were only calculated for abnormal ESEs. We performed statistical testing to compare the areas under the curve (AUCs) based on the DeLong method and computed 95\% confidence intervals [28]. We used a two-sided significance level of 0.05 and did not adjust for multiplicity. We conducted statistical analyses using SPSS version 26 (IBM Corporation, Armonk, NY, USA) and R version 4.0.2 (R Core Team, Vienna, Austria).

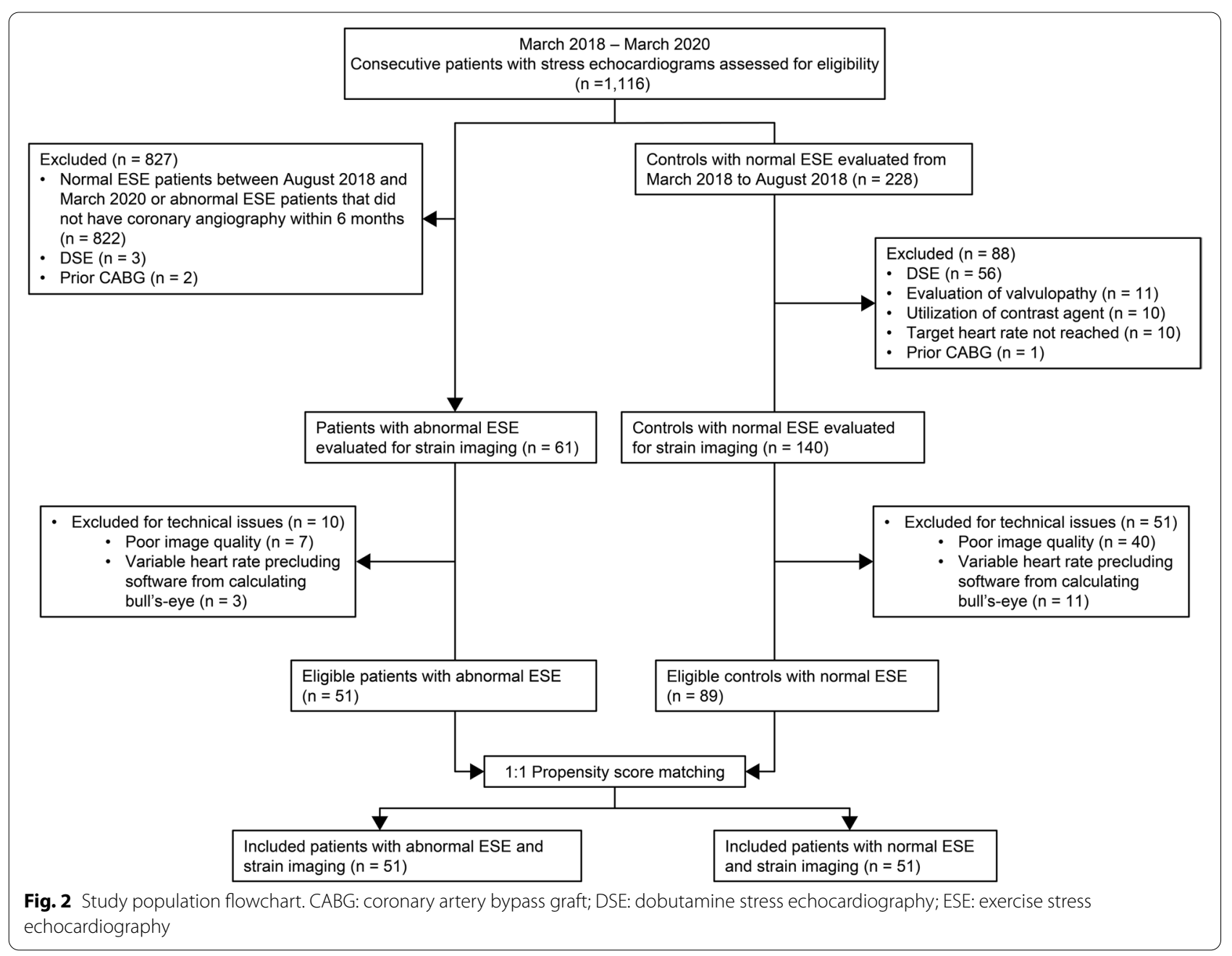


Table 1 Clinical characteristics and rest echocardiographic parameters in the propensity score matched patients

\begin{tabular}{|c|c|c|c|}
\hline Variables & $\begin{array}{l}\text { Abnormal ESE } \\
(n=51)\end{array}$ & $\begin{array}{l}\text { Normal ESE } \\
(n=51)\end{array}$ & $p$-values \\
\hline \multicolumn{4}{|l|}{ Clinical characteristics } \\
\hline Age (years) - mean (SD) & $66.4(8.7)$ & $65.1(9.4)$ & 0.49 \\
\hline Female sex - no. (\%) & $20(39.2)$ & $18(35.3)$ & 0.84 \\
\hline Hypercholesterolemia - no. (\%) & $31(60.8)$ & $27(53.0)$ & 0.55 \\
\hline Hypertension - no. (\%) & $31(60.8)$ & $26(51.0)$ & 0.43 \\
\hline Diabetes mellitus - no. (\%) & $12(23.5)$ & $9(17.7)$ & 0.63 \\
\hline Current smoker - no. (\%) & $14(27.5)$ & $13(25.5)$ & 1.00 \\
\hline Prior hemodynamically significant CAD ${ }^{\mathrm{a}}$ - no. (\%) & $13(25.5)$ & $14(27.5)$ & 1.00 \\
\hline \multicolumn{4}{|l|}{ Medications at time of ESE } \\
\hline Beta-blocker - no. (\%) & $12(23.5)$ & $10(19.6)$ & 0.81 \\
\hline ACE inhibitor/ARB - no. (\%) & $25(49.0)$ & $18(35.3)$ & 0.23 \\
\hline Calcium channel blocker - no. (\%) & $15(29.4)$ & $6(11.8)$ & 0.05 \\
\hline Long-acting nitrate - no. (\%) & $3(5.9)$ & $3(5.9)$ & 1.00 \\
\hline \multicolumn{4}{|l|}{ ESE parameters at rest } \\
\hline Left ventricular ejection fraction at rest - median (IQR) & $55.0(55.0-60.0)$ & $55.0(55.0-60.0)$ & 0.77 \\
\hline Presence of $\geq 1$ regional wall motion abnormalities at rest - no. (\%) & $15(29.4 \%)$ & $4(7.8 \%)$ & 0.005 \\
\hline Wall motion score index at rest - median (IQR) & $1.0(1.0-1.1)$ & $1.0(1.0-1.0)$ & 0.008 \\
\hline
\end{tabular}

$A C E$ Angiotensin-converting enzyme, $A R B$ angiotensin receptor blocker, $C A D$ coronary artery disease, ESE exercise stress echocardiography, SD standard deviation

${ }^{a} \mathrm{CAD}$ required a diagnosis by coronary angiogram and subsequent complete revascularization by percutaneous coronary intervention

\section{Results}

We excluded 61 patients from the STE analysis from 201 (30.3\%) eligible ESE due to technical issues; 47 (23.4\%) because of suboptimal image quality and 14 (7.0\%) because the heart rate was too variable between apical views precluding the software from calculating

Table 2 Treadmill ESE parameters at peak stress the bull's-eye (Fig. 2). Of note, STE analysis was possible for all patients at rest. Exclusions for suboptimal image quality was based on imaging at peak stress. After propensity score matching, there were 51 patients left in each group. Small differences (standardized differences $\geq 0.1$ ) remained in terms of age,

\begin{tabular}{|c|c|c|c|}
\hline Variables & $\begin{array}{l}\text { Abnormal stress } \\
\text { echocardiogram } \\
(n=51)\end{array}$ & $\begin{array}{l}\text { Normal stress } \\
\text { echocardiogram } \\
\text { CAD }(n=51)\end{array}$ & $p$-values \\
\hline \multicolumn{4}{|l|}{ Stress EKG } \\
\hline Maximum heart rate achieved (bpm) - mean (SD) & $141.9(16.3)$ & $148.5(16.1)$ & 0.04 \\
\hline Percentage of maximum predicted heart rate (\%) - median (IQR) & $92.0(88.0-99.0)$ & $94.0(90.0-101.0)$ & 0.08 \\
\hline Maximum systolic blood pressure (mmHg) - mean (SD) & $169.4(27.2)$ & $174.4(27.4)$ & 0.36 \\
\hline Abnormal blood pressure response ${ }^{\mathrm{a}}-$ no. $(\%)$ & $7(13.7)$ & $3(5.9)$ & 0.18 \\
\hline Positive EKG for ischemia during the stress test - no. (\%) & $35(68.6)$ & $27(52.9)$ & 0.11 \\
\hline Clinically positive stress test ${ }^{\mathrm{b}}-$ no. $(\%)$ & $22(43.1)$ & $3(5.9)$ & $<0.0001$ \\
\hline Maximum achieved METS - median (IQR) & $7.3(6.1-9.6)$ & $10.0(7.7-11.7)$ & $<0.0001$ \\
\hline Double product - mean (SD) & $23,884.7(4876.6)$ & $26,179.9(4705.6)$ & 0.02 \\
\hline \multicolumn{4}{|l|}{ Stress echocardiography } \\
\hline Absence of left ventricular ejection fraction increase at peak stress - no. (\%) & $31(60.8)$ & $0(0.0)$ & $<0.0001$ \\
\hline Wall motion score index at peak stress - median (IQR) & $1.4(1.2-1.5)$ & $1.0(1.0-1.0)$ & $<0.0001$ \\
\hline Predicted significant CAD in the LAD territory - no. (\%) & $33(64.7)$ & $0(0.0)$ & $<0.0001$ \\
\hline Predicted significant CAD in the non-LAD territory - no. (\%) & $25(49.0)$ & $0(0.0)$ & $<0.0001$ \\
\hline
\end{tabular}

CAD coronary artery disease, EKG electrocardiogram, IQR interquartile range, METS metabolic equivalents, SD standard deviation

${ }^{a}$ Defined as the failure to reach a systolic blood pressure of $120 \mathrm{mmHg}$ or to increase systolic blood pressure by more than $10 \mathrm{mmHg}$ at peak stress or $3 \mathrm{~min}$ after the cessation of the stress test

b Defined as angina or angina-like symptoms during stress treadmill stress test 
Table 3 Coronary angiogram characteristics

\begin{tabular}{|c|c|c|}
\hline \multicolumn{2}{|l|}{ Variables } & \multirow{2}{*}{$\begin{array}{l}\begin{array}{l}\text { Overall }^{\mathrm{a}} \\
(n=53)\end{array} \\
34.0(14.0-63.0)\end{array}$} \\
\hline \multicolumn{2}{|c|}{ Time between stress echocardiography and coronary angiogram (days) - median (IQR) } & \\
\hline \multirow{3}{*}{ Coronary dominance } & Right - no. (\%) & $44(83.0)$ \\
\hline & Left - no. (\%) & $5(9.4)$ \\
\hline & Co-dominance - no. (\%) & $4(7.5)$ \\
\hline Significant CAD overall - no. (\%) & & $35(66.0)$ \\
\hline Significant CAD in the LAD territory - no. (\%) & & $29(54.7)$ \\
\hline Significant CAD in the non-LAD territory - no. (\%) & & $30(56.6)$ \\
\hline Significant CAD in the Cx territory - no. (\%) & & $20(37.7)$ \\
\hline Significant CAD in RC the territory - no. (\%) & & $20(37.7)$ \\
\hline
\end{tabular}

$C A D$ coronary artery disease, $C X$ circumflex artery, IQR interquartile range, $L A D$ left anterior descending artery, $L M$ left main artery, $R C$ right coronary artery

a Patients who underwent coronary angiogram are comprised of the 51 patients with abnormal treadmill stress echocardiograms and two patients with normal treadmill stress echocardiograms

hypercholesterolemia, hypertension, and diabetes, but none were statistically significant (Table $\mathrm{S} 1$ ). The mean age of patients with abnormal ESE and normal ESE were 66.4 (39.2\% female) and 65.1 years (35.3\% female), respectively (Table 1 ).

While both groups had similar baseline left ventricular ejection fractions and achieved comparable systolic blood pressures and target heart rates, the patients with positive ESE had more WMA at rest, attained lower metabolic equivalent (METS) levels and double product levels (Table 2). In the abnormal ESE group, 33 patients (64.7\%) had significant CAD on angiography (Table 3 ). In the 1-year follow-up of the normal ESE group, 2 patients (3.9\%) required revascularization. One patient had persisting unequivocal symptoms and underwent a positive nuclear myocardial perfusion imaging study with subsequent percutaneous revascularization, while the other had typical angina and underwent percutaneous revascularization directly. There were no deaths in the propensity score matched normal ESE group.

The interobserver reliability of STE is presented in Table 4. Overall, we demonstrated good-to-excellent interobserver reliability for the GLS as well as for the STE for the apical segments as demonstrated by the intraclass correlation coefficient. Bland-Altman plots are shown in the Supplementary material (Figures S1-S4).

For the overall examination, the utilization of GLS alone at stress $(\mathrm{AUC}=0.69$ vs. $0.84, p=0.006)$ and the delta GLS (AUC $=0.69$ vs. $0.84, p=0.01$ ) resulted in inferior discrimination compared to visual assessment alone (Table 5). When considering normal ESE as negative and using STE to reclassify abnormal ESE, this combination was superior to visual assessment alone for the overall examination $(\mathrm{AUC}=0.89 \mathrm{vs}$. 0.84 , $p=0.025$ ), for the non-LAD territory (AUC $=0.83$ vs. $0.70, p=0.006$ ), but not for the LAD territory (AUC $=0.79$ vs. $0.73, p=0.11$ ) (Figs. 3,4 and 5 ). The sensitivity, specificity, negative predictive, and positive predictive values are shown in Table 5. Finally, we compared the strain characteristics of patients with significant CAD to those without significant CAD to demonstrate the increased variability and tendency toward larger differences between stress and rest values at the apex (Table S2).

\section{Discussion}

Our findings demonstrate that STE post-treadmill ESE is relatively feasible in patients who do not require a contrast agent, has good-to-excellent interobserver reliability between non-expert operators, and may provide reclassification value for patients with false-positive ESE. Furthermore, we showed that utilizing the STE difference between the apical segments at peak stress and baseline,

Table 4 Interobserver variability for strain imaging

\begin{tabular}{|c|c|c|c|c|}
\hline$r$ & Bias & LOA & $\begin{array}{l}\text { \% mean relative } \\
\text { difference }\end{array}$ & $\begin{array}{l}\text { ICC } \\
\text { (mean } \\
\text { rating) }\end{array}$ \\
\hline \multicolumn{5}{|c|}{ Baseline GLS } \\
\hline 0.79 & 0.63 & \pm 3.81 & $-4 \%$ & 0.87 \\
\hline \multicolumn{5}{|c|}{ Baseline apical segments (segments 13-17) } \\
\hline 0.74 & 1.15 & \pm 6.74 & $-6 \%$ & 0.84 \\
\hline \multicolumn{5}{|c|}{ Peak stress GLS } \\
\hline 0.85 & 0.09 & \pm 4.12 & $-1.47 \%$ & 0.92 \\
\hline \multicolumn{5}{|c|}{ Peak stress apical segments (segments 13-17) } \\
\hline 0.81 & -0.78 & \pm 9.24 & $4.19 \%$ & 0.89 \\
\hline
\end{tabular}

ICC intra-class correlation coefficient, GLS global longitudinal strain, LOA limit of agreement $( \pm 1.96$ standard deviations $) ; \%$ mean relative difference $=(100 \mathrm{x}$ (measure 1 - measure 2))/(measure 1) 
Table 5 Diagnostic accuracy by visual and strain imaging for detecting significant coronary stenoses

\begin{tabular}{|c|c|c|c|c|c|c|c|c|}
\hline & $\begin{array}{l}\text { Sensitivity } \\
\text { (\%) }\end{array}$ & $\begin{array}{l}\text { Specificity } \\
\text { (\%) }\end{array}$ & $\begin{array}{l}\text { PPV } \\
\text { (\%) }\end{array}$ & $\begin{array}{l}\text { NPV } \\
\text { (\%) }\end{array}$ & $\begin{array}{l}\text { Accuracy } \\
\text { (\%) }\end{array}$ & Optimal cut-off & $\begin{array}{l}\text { AUC } \\
(95 \% \mathrm{Cl})\end{array}$ & $p$-value \\
\hline \multicolumn{9}{|l|}{ Visual assessment } \\
\hline Overall examination & 94.3 & 73.1 & 64.7 & 96.1 & 80.4 & N/A & $0.84(0.73-0.94)$ & Ref. \\
\hline $\mathrm{LAD}$ & 65.5 & 80.8 & 57.6 & 85.5 & 76.5 & N/A & $0.73(0.66-0.80)$ & Ref. \\
\hline Non-LAD & 53.3 & 87.5 & 64.0 & 81.8 & 77.5 & N/A & $0.70(0.66-0.75)$ & Ref. \\
\hline \multicolumn{9}{|l|}{ Stress GLS } \\
\hline Overall examination & 68.6 & 68.7 & 53.3 & 80.7 & 68.7 & -19.9 & $0.69(0.58-0.80)$ & 0.006 \\
\hline $\mathrm{LAD}$ & 72.4 & 69.9 & 48.8 & 86.4 & 70.6 & -21.2 & $0.75(0.64-0.86)$ & 0.63 \\
\hline Non-LAD & 56.7 & 65.3 & 40.5 & 78.3 & 62.8 & -15.0 & $0.60(0.47-0.72)$ & 0.09 \\
\hline \multicolumn{9}{|l|}{ Delta GLS } \\
\hline Overall examination & 42.9 & 92.5 & 75.0 & 75.6 & 75.5 & 4.1 & $0.69(0.57-0.80)$ & 0.01 \\
\hline LAD & 72.4 & 76.7 & 55.3 & 87.5 & 75.5 & 1.1 & $0.75(0.63-0.87)$ & 0.67 \\
\hline Non-LAD & 43.3 & 80.6 & 48.2 & 77.3 & 69.6 & 3.7 & $0.60(0.47-0.72)$ & 0.10 \\
\hline \multicolumn{9}{|l|}{ Stress apical segment STE } \\
\hline Overall examination (S13-17) & 51.4 & 89.6 & 72.0 & 77.9 & 76.5 & -21.2 & $0.76(0.65-0.86)$ & 0.15 \\
\hline LAD (segment 17) & 62.1 & 86.3 & 64.3 & 85.1 & 79.4 & -21.8 & $0.78(0.67-0.89)$ & 0.23 \\
\hline Non-LAD (S15 and 16) & 66.7 & 77.8 & 55.6 & 84.9 & 74.5 & -22.8 & $0.77(0.67-0.87)$ & 0.38 \\
\hline \multicolumn{9}{|l|}{ Delta apical segment STE } \\
\hline Overall examination (S13-17) & 65.7 & 82.1 & 65.7 & 82.1 & 76.5 & 2.1 & $0.76(0.65-0.86)$ & 0.16 \\
\hline LAD (segment 17) & 72.4 & 76.7 & 55.3 & 87.5 & 75.5 & 1.8 & $0.78(0.66-0.89)$ & 0.31 \\
\hline Non-LAD (S15 and 16) & 66.7 & 77.8 & 55.6 & 84.9 & 74.5 & 1.9 & $0.76(0.65-0.86)$ & 0.49 \\
\hline \multicolumn{9}{|c|}{ Visual assessment with delta apical segment strain STE if visual assessment positive ${ }^{a}$} \\
\hline Overall examination & 94.3 & 77.6 & 68.8 & 96.3 & 83.3 & 4.8 & $0.89(0.82-0.96)$ & 0.025 \\
\hline $\mathrm{LAD}$ & 69.0 & 87.7 & 69.0 & 87.7 & 82.4 & 4.8 & $0.79(0.67-0.90)$ & 0.11 \\
\hline Non-LAD & 73.3 & 87.5 & 71.0 & 88.7 & 83.3 & -2.8 & $0.83(0.73-0.92)$ & 0.006 \\
\hline
\end{tabular}

AUC area under the curve, GLS global longitudinal strain, PPV positive predictive value, NPV negative predictive value, S segments, STE two-dimensional speckletracking echocardiography

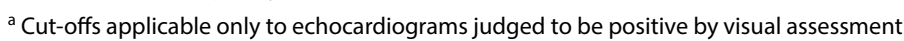

in addition to visual assessment, maximized the diagnostic yield.

The sensitivity, specificity, and accuracy values of $94.3 \%, 73.1 \%$, and $80.4 \%$ for the overall examination for visual assessment alone in our study are comparable to published estimates [1]. The high negative predictive value (96.1\%) supports the excellent prognostic value for symptomatic obstructive CAD of a negative ESE [29]. However, the visual assessment sensitivity must be interpreted with caution because of the test verification bias and because the study sample was created by propensity score matching.

The combination of visual assessment and STE did lead to a slightly increased discrimination capacity. Compared to visual assessment alone, the combination of visual assessment and STE seemed to improve the accuracy of the detection of non-LAD stenoses in a clinically meaningful manner ( $77.5 \%$ vs. $83.3 \%$ ). Of note, $30.3 \%$ of eligible patients who did not require a contrast agent were excluded, a higher proportion than typically displayed in DSE studies [1]. This significant exclusion of patients displayed the difficulty in obtaining high-quality images with little heart rate variability suitable for strain analysis in the ninety-second post-exercise window. However, our non-expert interobserver reliability was comparable to previous data of expert operators reflecting the intuitiveness of STE [30].

Our study is concordant with previous DSE studies, demonstrating that various strain measures may provide an incremental diagnostic yield to visual assessment [7, 31]. However, this has not been shown consistently by other authors [30]. Previous studies with promising results have utilized GLS $[1,32]$. In our study, the use of peak stress GLS or the use of the difference between peak stress GLS and baseline GLS resulted in inferior discrimination than visual assessment for the overall examination. 


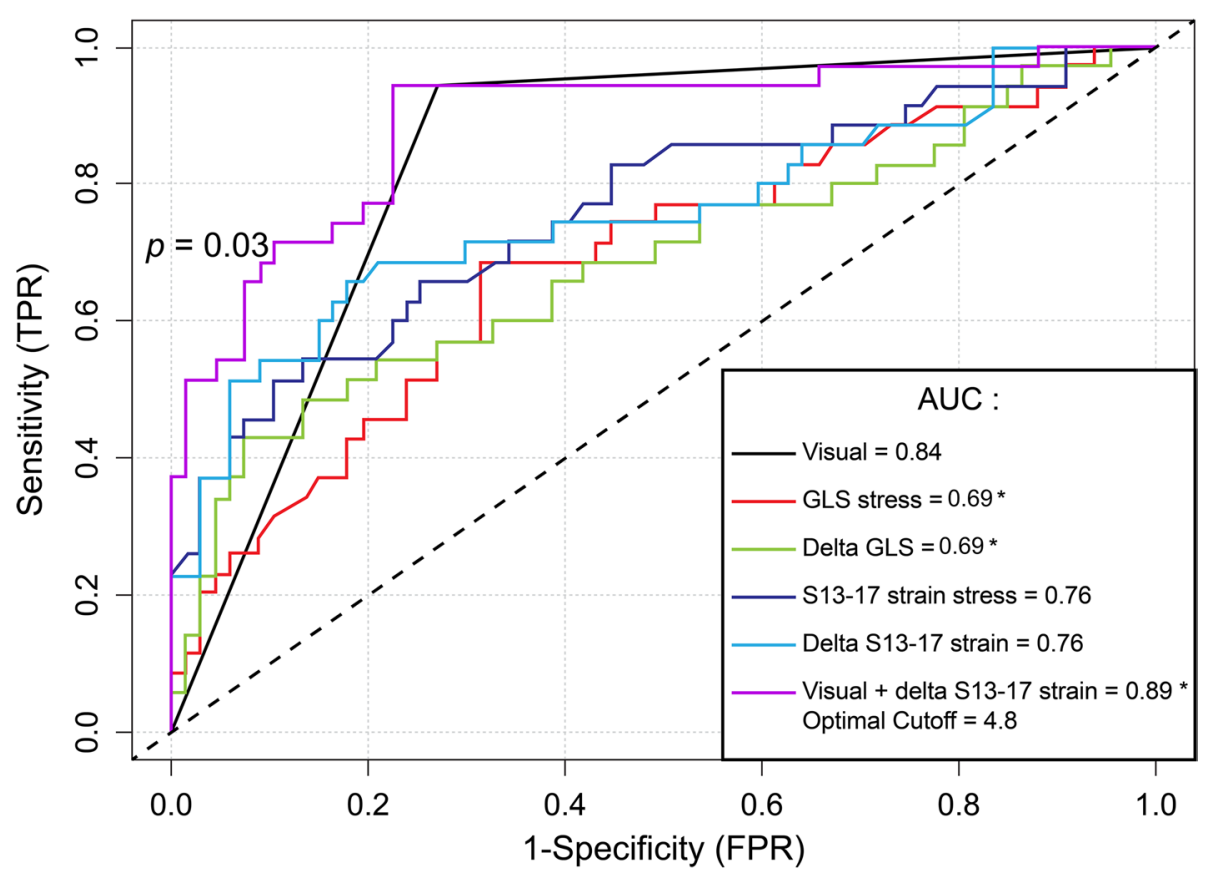

Fig. 3 Receiver operator characteristic curve for the detection of significant coronary artery disease for the overall examination. ${ }^{*}$ Denotes $p<0.05$ compared to visual assessment

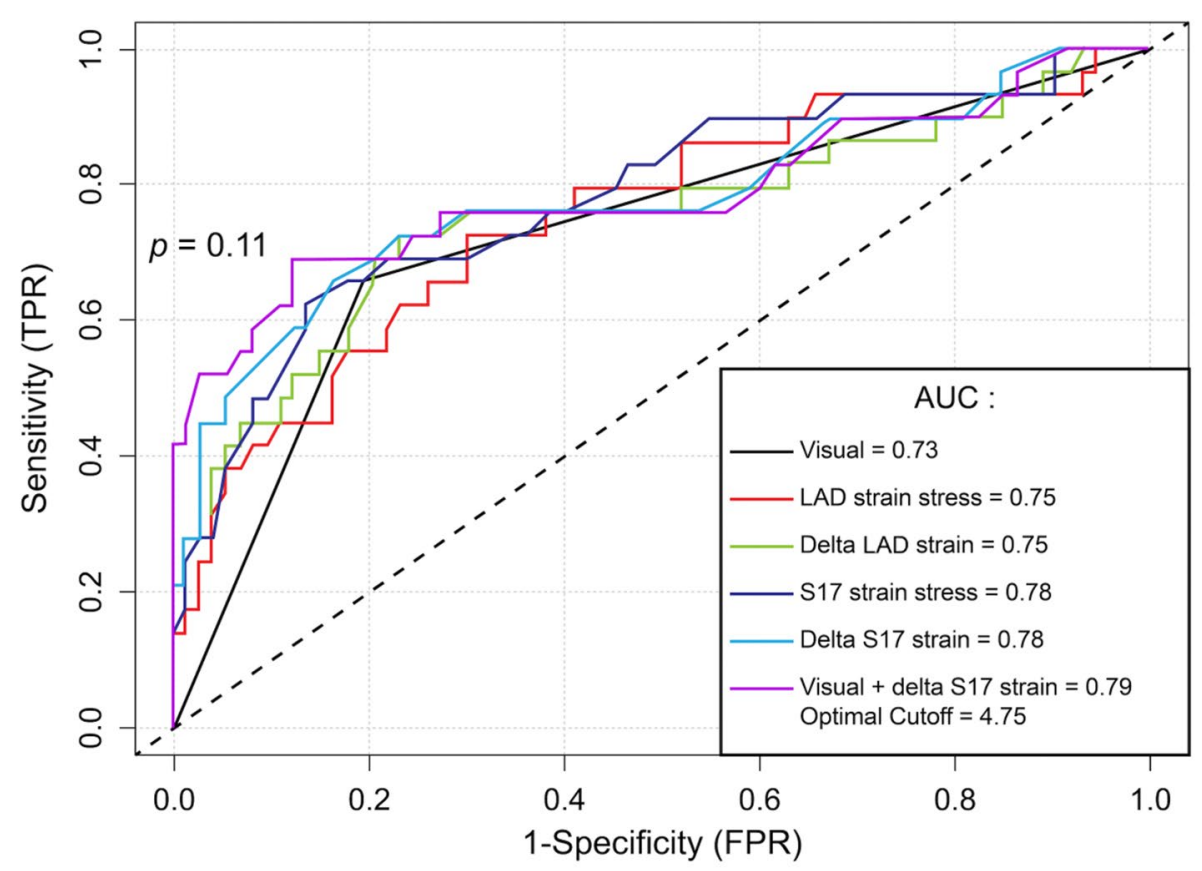

Fig. 4 Receiver operator characteristic curve for the detection of significant coronary artery disease in the left anterior descending artery territory. *Denotes $p<0.05$ compared to visual assessment 


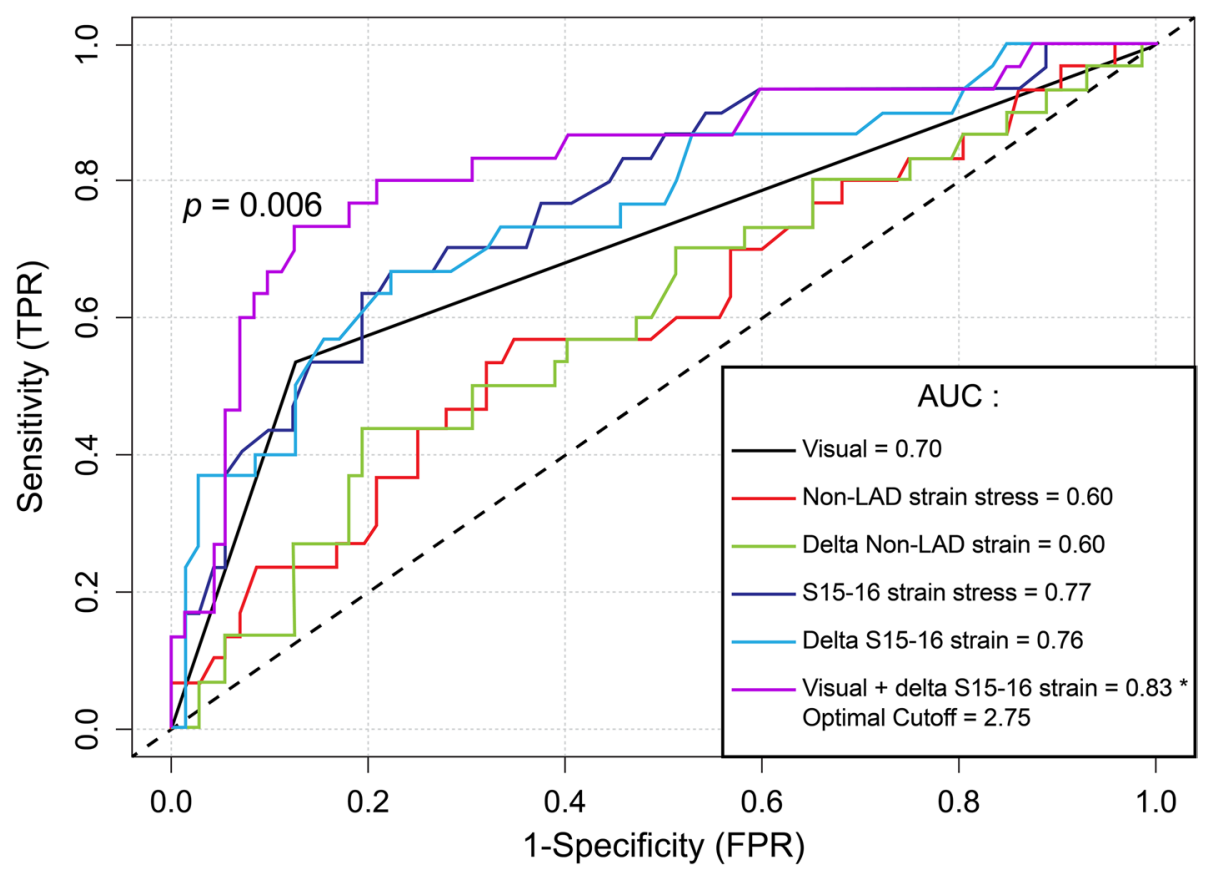

Fig. 5 Receiver operator characteristic for the detection of significant coronary artery disease in the non-left anterior descending artery territory. *Denotes $p<0.05$ compared to visual assessment

We hypothesize that our adjustment for confounding by selecting non-healthy controls may have attenuated the value of GLS for detecting significant CAD. Furthermore, a previous study that combined treadmill ESE and strain imaging did not utilize peak stress images and did not demonstrate conclusive results [10].

Our findings are in agreement with prior research that showed the utility of the apex (segment 17) for diagnosing LAD CAD disease, as well as the applicability of calculating the difference between peak and stress values in patients with WMA [6]. As ESE can provide important prognostic information by reproducing the physiological response with exercise, it is generally considered the test of choice over DSE [1]. Further studies are needed to validate the additional diagnostic value of strain imaging in treadmill ESE.

\section{Study limitations}

The main limitation of our study was the absence of systematic coronary angiograms for all eligible patients. This was not feasible due to the study's retrospective nature. While we could not evaluate CAD in all patients with negative ESE, the one-year follow-up allowed us to examine for clinically driven diagnoses of coronary stenoses. For this reason, our diagnostic parameters must be interpreted with caution and viewed as hypothesis-generating. Our study may provide investigators with pilot data to conduct a prospective study with systematic angiograms to provide true estimates of speckle-tracking sensitivity in the post treadmill exercice context. Second, we could not complete STE in patients who required contrast and did not have optimal images, thus limiting our study's generalizability. Third, the visual assessment of the ESEs was only done by one single echocardiographer. Fourth, STE evaluations were performed by two non-expert operators. Finally, we did not achieve perfect correction of characteristics imbalance between the two groups of patients with standardized differences above 0.1 in some covariates. However, achieving standardized differences below 0.25 may be reasonable given our modest sample size [26].

\section{Conclusions}

Two-dimensional speckle-tracking of the apical segments combined with treadmill ESE is relatively feasible in patients who do not require contrast, has good interobserver reliability, and may provide incremental diagnostic value for reclassifying false-positive ESE. Future prospective studies of ESE with two-dimensional speckle-tracking with larger sample sizes and coronary angiograms for all study participants are needed to confirm our findings. 


\section{Abbreviations}

AUC: Area under the curve; CAD: Coronary artery disease; DSE: Dobutamine stress echocardiography; ESE: Exercise stress echocardiography; GLS: Global longitudinal strain; LAD: Left anterior descending artery; LS: Longitudinal strain; STE: Two-dimensional speckle-tracking echocardiography; ROC: Receiver operating characteristic; WMA: Wall motion abnormality.

\section{Supplementary Information}

The online version contains supplementary material available at https://doi. org/10.1186/s12947-021-00259-w.

Additional file 1: Table S1. Clinical characteristics before and after propensity score matching Table S2. Strain characteristics of patients with significant CAD on coronary angiogram compared to patients without significant CAD on angiogram/patients with visually normal ESE. Figure S1. Bland-Altman plot for baseline global longitudinal strain. Figure S2. Bland-Altman plot for peak stress global longitudinal strain. Figure S3. Bland-Altmann plot for baseline strain of the apical segments (segments 13-17). Figure S4. Bland-Altmann plot for peak stress strain of the apical segments (segments 13-17).

\section{Acknowledgements}

The authors would like to acknowledge the sonographers Stéphanie Paquette, Hélène Plante, Natalie Rodrigue, Marie-Michèle Rondeau, and Patrice Trottier for their diligence throughout the study. We could not have completed the study without their expertise and hard work.

\section{Authors' contributions}

$M A D, E C$, and $B E$ designed the study. EC screened the echocardiograms. MAD and GF performed the strain analysis. PF, MCB, DHD, SL, WMM, and BE performed the visual interpretation of the echocardiograms. SLP and MAD performed the statistical analysis. MAD drafted the manuscript. TH, ELC, and MN participated in the revision of the manuscript. All authors read and approved the final manuscript.

\section{Funding}

The study was funded by Sherbrooke University and the Sherbrooke University Hospital Center (CHUS).

\section{Availability of data and materials}

The data that support the study findings are available from the corresponding author upon reasonable request.

\section{Declarations}

\section{Ethics approval and consent to participate}

The study was approved by the Sherbrooke University Hospital Research Center (CRCHUS) ethics committee, and individual patient consent was not required.

\section{Consent for publication \\ Not applicable.}

\section{Competing interests}

The authors have no competing interests to disclose.

\section{Author details}

${ }^{1}$ Division of Cardiology, Department of Medicine, Sherbrooke University Hospital Center (CHUS), 3001, 12e Avenue Nord, Sherbrooke, QC J1H 5N4, Canada. ${ }^{2}$ Harvard T.H. Chan School of Public Health, Boston, MA, USA. ${ }^{3}$ McGill Health University Center, Montreal, QC, Canada. ${ }^{4}$ Sherbrooke University Hospital Research Center (CRCHUS), Sherbrooke, QC, Canada.

Received: 24 May 2021 Accepted: 13 July 2021

Published online: 23 July 2021

\section{References}

1. Pellikka PA, Arruda-OIson A, Chaudhry FA, Chen MH, Marshall JE, Porter TR, Sawada SG. Guidelines for Performance, Interpretation, and Application of Stress Echocardiography in Ischemic Heart Disease: From the American Society of Echocardiography. J Am Soc Echocardiogr. 2020;33(1):1-41.

2. Pellikka PA, Nagueh SF, Elhendy AA, Kuehl CA, Sawada SG. American Society of E: American Society of Echocardiography recommendations for performance, interpretation, and application of stress echocardiography. J Am Soc Echocardiogr. 2007;20(9):1021-41.

3. Knuuti J, Ballo H, Juarez-Orozco LE, Saraste A, Kolh P, Rutjes AWS, Juni P, Windecker S, Bax JJ, Wijns W. The performance of non-invasive tests to rule-in and rule-out significant coronary artery stenosis in patients with stable angina: a meta-analysis focused on post-test disease probability. Eur Heart J. 2018;39(35):3322-30.

4. Guerreiro RA, Fazendas P, Pereira AR, Marques A, Pais J, Alegria S, Congo KH, Gomes AC, Carvalho J, Morgado G, et al. Clinical and Echocardiographic Characterization of False-Positive Results from Stress Echocardiography. J Cardiovasc Imaging. 2020;28(2):123-33.

5. Voigt JU, Pedrizzetti G, Lysyansky P, Marwick TH, Houle H, Baumann R, Pedri S, Ito Y, Abe Y, Metz S, et al. Definitions for a common standard for 2D speckle tracking echocardiography: consensus document of the EACVI/ASE/Industry Task Force to standardize deformation imaging. Eur Heart J Cardiovasc Imaging. 2015;16(1):1-11.

6. Joyce E, Hoogslag GE, Al Amri I, Debonnaire P, Katsanos S, Bax JJ, Delgado V, Marsan NA. Quantitative Dobutamine Stress Echocardiography Using Speckle-Tracking Analysis versus Conventional Visual Analysis for Detection of Significant Coronary Artery Disease after ST-Segment Elevation Myocardial Infarction. J Am Soc Echocardiogr. 2015;28(12):1379-89.

7. Uusitalo V, Luotolahti M, Pietila M, Wendelin-Saarenhovi M, Hartiala J, Saraste M, Knuuti J, Saraste A. Two-Dimensional Speckle-Tracking during Dobutamine Stress Echocardiography in the Detection of Myocardial Ischemia in Patients with Suspected Coronary Artery Disease. J Am Soc Echocardiogr. 2016;29(5):470-9.

8. Biering-Sorensen T, Hoffmann S, Mogelvang R, Zeeberg Iversen A, Galatius S, Fritz-Hansen T, Bech J, Jensen JS. Myocardial strain analysis by 2 -dimensional speckle tracking echocardiography improves diagnostics of coronary artery stenosis in stable angina pectoris. Circ Cardiovasc Imaging. 2014;7(1):58-65.

9. Larsen AH, Clemmensen TS, Wiggers H, Poulsen SH. Left ventricular myocardial contractile reserve during exercise stress in healthy adults: a two-dimensional speckle-tracking echocardiographic study. J Am Soc Echocardiogr. 2018;31(10):1116-26.

10. Takagi T, Takagi A, Yoshikawa J. Detection of coronary artery disease using delayed strain imaging at 5 min after the termination of exercise stress: head to head comparison with conventional treadmill stress echocardiography. J Cardiol. 2010;55(1):41-8.

11. von Scheidt F, Kiesler V, Kaestner M, Bride P, Kramer J, Apitz C. Left Ventricular Strain and Strain Rate during Submaximal Semisupine Bicycle Exercise Stress Echocardiography in Healthy Adolescents and Young Adults: Systematic Protocol and Reference Values. J Am Soc Echocardiogr. 2020;33(7):848-57.

12. Bossuyt PM, Reitsma JB, Bruns DE, Gatsonis CA, Glasziou PP, Irwig L, Lijmer JG, Moher D, Rennie D, de Vet HC, et al. STARD 2015: an updated list of essential items for reporting diagnostic accuracy studies. BMJ. 2015:351:h5527.

13. Aggeli C, Lagoudakou S, Felekos I, Panagopoulou V, Kastellanos S, Toutouzas K, Roussakis G, Tousoulis D. Two-dimensional speckle tracking for the assessment of coronary artery disease during dobutamine stress echo: clinical tool or merely research method. Cardiovasc Ultrasound. 2015;13:43.

14. Cusma-Piccione M, Zito C, Oreto L, D'Angelo M, Tripepi S, Di Bella G, Todaro MC, Oreto G, Khandheria BK, Carerj S. Longitudinal Strain by Automated Function Imaging Detects Single-Vessel Coronary Artery Disease in Patients Undergoing Dipyridamole Stress Echocardiography. J Am Soc Echocardiogr. 2015;28(10):1214-21.

15. Hwang HJ, Lee HM, Yang $H$, Lee JL, Pak HY, Park CB, Jin ES, Cho JM, Kim CJ, Sohn IS. The value of assessing myocardial deformation at recovery after dobutamine stress echocardiography. J Cardiovasc Ultrasound. 2014:22(3):127-33. 
16. Nakai H, Takeuchi M, Nishikage T, Lang RM, Otsuji Y. Subclinical left ventricular dysfunction in asymptomatic diabetic patients assessed by two-dimensional speckle tracking echocardiography: correlation with diabetic duration. Eur J Echocardiogr. 2009;10(8):926-32.

17. Navarini S, Bellsham-Revell H, Chubb H, Gu H, Sinha MD, Simpson JM. Myocardial Deformation Measured by 3-Dimensional Speckle Tracking in Children and Adolescents With Systemic Arterial Hypertension. Hypertension. 2017;70(6):1142-7.

18. Sugimoto T, Dulgheru R, Bernard A, Ilardi F, Contu L, Addetia K, Caballero L, Akhaladze N, Athanassopoulos GD, Barone D, et al. Echocardiographic reference ranges for normal left ventricular 2D strain: results from the EACVI NORRE study. Eur Heart J Cardiovasc Imaging. 2017;18(8):833-40.

19. Yaman B, Akpinar O, Cerit L, Kemal HS, Usalp S, Yuksek U, Acikgoz E, Coskun $U$, Duygu $\mathrm{H}$. Effects of chronic cigarette smoking on myocardial deformation parameters by two-dimensional speckle tracking echocardiography. Echocardiography. 2019;36(11):2026-32.

20. Burroughs Pena M, Swett K, Schneiderman N, Spevack DM, Ponce SG, Talavera GA, Kansal MM, Daviglus ML, Cai J, Hurwitz BE, et al. Cardiac structure and function with and without metabolic syndrome: the Echocardiographic Study of Latinos (Echo-SOL). BMJ Open Diabetes Res Care. 2018;6(1).

21. Rumbinaite E, Zaliaduonyte-Peksiene D, Lapinskas T, Zvirblyte R, Karuzas A, Jonauskiene I, Viezelis M, Ceponiene I, Gustiene O, Slapikas R, et al. Early and late diastolic strain rate vs global longitudinal strain at rest and during dobutamine stress for the assessment of significant coronary artery stenosis in patients with a moderate and high probability of coronary artery disease. Echocardiography. 2016;33(10):1512-22.

22. Wierzbowska-Drabik K, Plewka M, Kasprzak JD. Variability of longitudinal strain in left ventricular segments supplied by non-stenosed coronary artery: insights from speckle tracking analysis of dobutamine stress echocardiograms in patients with high coronary risk profile. Arch Med Sci. 2017;13(1):82-92.

23. Fletcher GF, Ades PA, Kligfield P, Arena R, Balady GJ, Bittner VA, Coke LA, Fleg JL, Forman DE, Gerber TC, et al. Exercise standards for testing and training: a scientific statement from the American Heart Association. Circulation. 2013;128(8):873-934.

24. Neumann FJ, Sousa-Uva M, Ahlsson A, Alfonso F, Banning AP, Benedetto U, Byrne RA, Collet JP, Falk V, Head SJ, et al. 2018 ESC/EACTS Guidelines on myocardial revascularization. Eur Heart J. 2019;40(2):87-165.
25. Austin PC. A comparison of 12 algorithms for matching on the propensity score. Stat Med. 2014;33(6):1057-69.

26. Austin PC. Balance diagnostics for comparing the distribution of baseline covariates between treatment groups in propensity-score matched samples. Stat Med. 2009;28(25):3083-107.

27. Liljequist D, Elfving B, Skavberg Roaldsen K. Intraclass correlation - A discussion and demonstration of basic features. PLoS One. 2019;14(7)

28. DeLong E, DeLong D, Clarke-Pearson D. Comparing the Areas Under Two or More Correlated Receiver Operating Characteristics Curves: A Nonparametric Approach. Biometrics. 1988;44:837-45.

29. Cortigiani L, Urluescu ML, Coltelli M, Carpeggiani C, Bovenzi F, Picano E. Apparent declining prognostic value of a negative stress echocardiography based on regional wall motion abnormalities in patients with normal resting left ventricular function due to the changing referral profile of the population under study. Circ Cardiovasc Imaging. 2019;12(6).

30. Yang LT, Kado Y, Nagata Y, Otani K, Otsuji Y, Takeuchi M. Strain Imaging with a Bull's-Eye Map for Detecting Significant Coronary Stenosis during Dobutamine Stress Echocardiography. J Am Soc Echocardiogr. 2017;30(2):159-67.

31. Ng AC, Sitges M, Pham PN, da Tran T, Delgado V, Bertini M, Nucifora G, Vidaic J, Allman C, Holman ER, et al. Incremental value of 2-dimensional speckle tracking strain imaging to wall motion analysis for detection of coronary artery disease in patients undergoing dobutamine stress echocardiography. Am Heart J. 2009;158(5):836-44.

32. Roushdy A. Abou El Seoud Y, Abd Elrahman M, Wadeaa B, Eletriby A, Abd El Salam Z: The additional utility of two-dimensional strain in detection of coronary artery disease presence and localization in patients undergoing dobutamine stress echocardiogram. Echocardiography. 2017;34(7):1010-9.

\section{Publisher's Note}

Springer Nature remains neutral with regard to jurisdictional claims in published maps and institutional affiliations.
Ready to submit your research? Choose BMC and benefit from:

- fast, convenient online submission

- thorough peer review by experienced researchers in your field

- rapid publication on acceptance

- support for research data, including large and complex data types

- gold Open Access which fosters wider collaboration and increased citations

- maximum visibility for your research: over $100 \mathrm{M}$ website views per year

At BMC, research is always in progress.

Learn more biomedcentral.com/submissions 